\title{
Receptivity coefficients of vortex-vibrational type at excitation of 3D Tollmien-Schlichting waves in a boundary layer on a swept wing
}

Cite as: AIP Conference Proceedings 2125, 030044 (2019); https://doi.org/10.1063/1.5117426

Published Online: 26 July 2019

V. I. Borodulin, A. V. Ivanov, Y. S. Kachanov, D. A. Mischenko, R. Örlü, A. Hanifi, and S. Hein

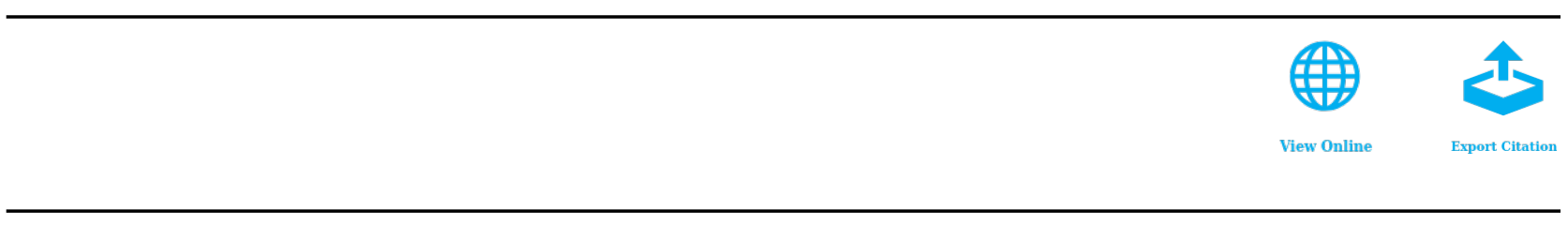

\section{Conference Proceedings}

Get $30 \%$ off all print proceedings!
Enter Promotion Code PDF-30 at checkout

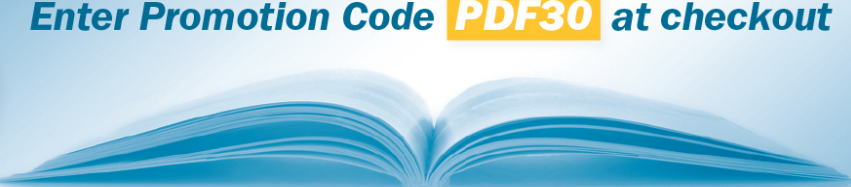




\title{
Receptivity Coefficients of Vortex-Vibrational Type at Excitation of 3D Tollmien-Schlichting Waves in a Boundary Layer on a Swept Wing
}

\author{
V. I. Borodulin, a), A. V. Ivanov ${ }^{1, \text { b) }}$, Y. S. Kachanov ${ }^{1, c)}$, D. A. Mischenko, d), \\ R. Örlü ${ }^{2, e)}$, A. Hanifi ${ }^{2, f)}$, and S. Hein ${ }^{3, g)}$ \\ ${ }^{1}$ Khristianovich Institute of Theoretical and Applied Mechanics SB RAS, \\ Institutskaya str., 4/1, Novosibirsk, 630090, Russia \\ ${ }^{2}$ KTH Royal Institute of Technology, Linné FLOW Centre, Dept. of Mechanics, \\ SE-100 44, Stockholm, Sweden \\ ${ }^{3}$ Institute of Aerodynamics and Flow Technology, DLR, \\ 37073, Göttingen, Germany \\ a)kachanov@itam.nsc.ru \\ b)bo@itam.nsc.ru \\ c)aniva101010@gmail.com \\ ${ }^{\mathrm{d})}$ Corresponding author: D.A.Mischenko@itam.nsc.ru \\ e)ramis@mech.kth.se \\ f)hanifi@kth.se

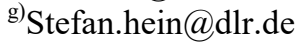

\begin{abstract}
The paper is devoted to the first results of an experimental quantitative study of the receptivity mechanism of a swept-wing laminar boundary layer related to scattering of $2 \mathrm{D}$ freestream vortices (with frequency $f_{\mathrm{v}}$ ) at $3 \mathrm{D}$ local surface vibrations (with frequency $f_{\mathrm{s}}$ ) resulting in an excitation of Tollmien-Schlichting (TS) waves (having combination frequencies $f_{+}=f_{\mathrm{s}}+f_{\mathrm{v}}$ and $f_{-}=f_{\mathrm{s}}-f_{\mathrm{v}}$ ). The experiments were carried out in a low-turbulence level wind tunnel on a highprecision experimental model of long-laminar-run swept airfoil (sweep angle of $35^{\circ}$ ) at a freestream speed of about $10 \mathrm{~m} / \mathrm{s}$. Controlled localized 3D surface vibrations and 2D freestream vortices were generated by special disturbance sources. Quantitative characteristics of the studied receptivity mechanism (receptivity coefficients) were estimated.
\end{abstract}

\section{INTRODUCTION}

In spite of a great number of theoretical investigations of various vortex receptivity mechanisms, the amount of quantitative experimental studies of excitation of instability waves by freestream vortices or due to the scattering of freestream vortices on surface nonuniformities is rather restricted.

For the first time the vortex receptivity problem was investigated experimentally in the 2D formulation [1-3]). It was found that 2D Tollmien-Schlichting (TS)-waves can be excited rather efficiently near the plate leading edge while further downstream the freestream vortices are not able to penetrate into the boundary layer. Later it was found experimentally [4] that the presence of surface roughness elements provides the possibility of a rather efficient localized excitation of 2D TS-waves.

Localized excitation of 3D TS-waves by freestream vortices in presence of surface roughness elements was investigated in experiments on a flat plate [5,6] and on an airfoil [7]. Estimates of the corresponding localized receptivity coefficients were obtained for the "vortex-vibrational" [5, 6] and "vortex-roughness" [7] receptivity mechanisms. Similar results were obtained later for a 3D, swept-wing boundary layer at excitation of cross-flow

High-Energy Processes in Condensed Matter (HEPCM 2019)

AIP Conf. Proc. 2125, 030044-1-030044-7; https://doi.org/10.1063/1.5117426

Published by AIP Publishing. 978-0-7354-1865-3/\$30.00 
(CF) instability modes by both vortex-roughness [8] and vortex-vibrational [9] localized receptivity mechanisms. The excitation of nonstationary Görtler instability modes due to scattering of freestream vortices on localized surface nonuniformities was investigated recently in experiments [10]. In the same period of time, several experimental studies were devoted also to various mechanisms of distributed excitation of instability modes (TS-wave, CF-modes, and Görtler vortices), both two- and three-dimensional ones, in 2D and 3D boundary layers. We abstain from reviewing these works for the sake brevity because the present paper is devoted to the localized receptivity mechanism.

The important point is that the problem of excitation of the TS-waves in three-dimensional, swept-wing boundary layers, especially on real swept airfoils, has never been studied experimentally for cases of either localized or distributed vortex receptivity. The present investigation aims to fill this gap.

\section{EXPERIMENTAL SETUP}

The experiments were carried out in the Minimum Turbulence Level (MTL) wind tunnel at KTH (Stockholm) in a 3D boundary layer of a high-precision experimental model. It represents a long-laminar-run airfoil section of a swept wing with chord length normal to the leading edge of $800 \mathrm{~mm}$ and sweep angle of 35 degrees equipped with sidewalls of special shape mounted at the ceiling and the floor of the test section [11]. The sidewalls served for satisfaction of the infinite-span swept-wing conditions (see Fig. 1). The wing was set up at an angle of attack of $+1.5^{\circ}$ and exposed to an incident flow velocity of about $10 \mathrm{~m} / \mathrm{s}$. As a result, the boundary layer under study was close to the Blasius one (but still three-dimensional) and was stable with respect to the CF-instability modes but unstable with respect to the 3D TS-waves.

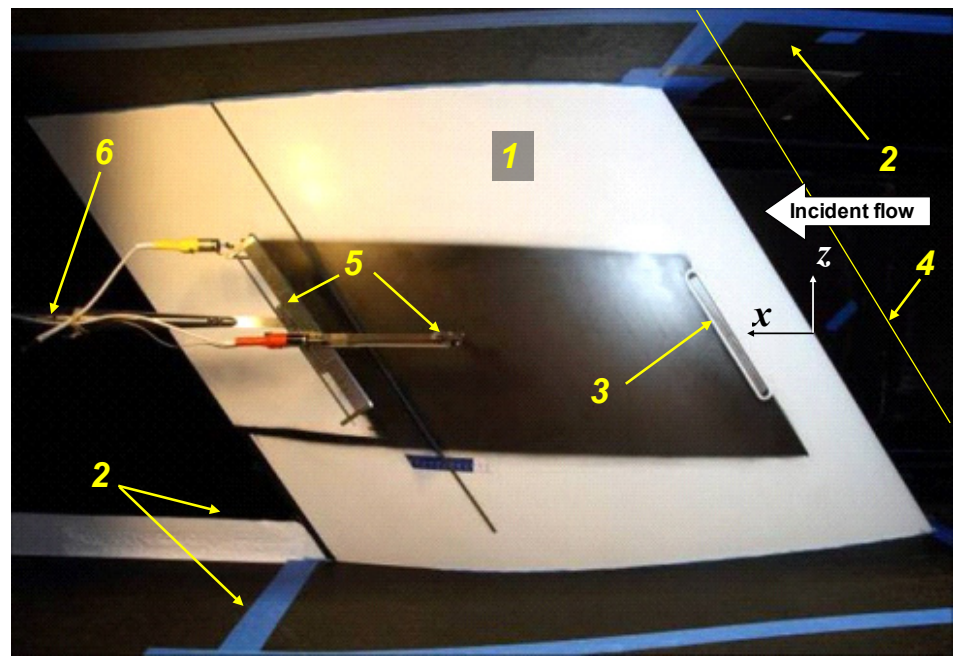

FIGURE 1. Experimental model in wind-tunnel test section. 1 - airfoil, 2 - sidewalls, 3 - surface disturbance source, 4 - vibrating wire, 5 - high-precision traverse mechanism "Komarik", 6 - sting of main traverse of MTL

Hot-wire measurements were carried out at fully controlled disturbance conditions. The source of localized 3D surface vibrations represented a spanwise oriented row of elastic circular membranes (with diameter of $6 \mathrm{~mm}$ and spacing of $8 \mathrm{~mm}$ ) mounted at chord position of $120 \mathrm{~mm}$. In the regimes of excitation described in the present paper, only one membrane of the source was turned on. It oscillated at frequency $f_{\mathrm{s}}$ with amplitude of about $100 \mu \mathrm{m}$ under action of rather weak pressure fluctuations, which were generated by a loudspeaker located outside of the windtunnel test section and connected with a chamber located under the membrane by a long plastic pipe. The shape of the surface vibrations was carefully measured by a high-precision laser displacement meter (optoNCDT-1605) in every studied regime (see Fig. 2, a). It was found that the shape of vibrations does not depend on their frequency and amplitude. The amplitude part of the Fourier spectrum of the surface vibrations is shown in Fig. 2, b. Controlled freestream perturbations of frequency $f_{\mathrm{v}}$ represented a $2 \mathrm{D}$ antisymmetric vortex street excited by a vibrating wire (having a diameter of $50 \mu \mathrm{m}$ ) tensioned parallel to the model leading edge not far upstream of it. The vortices traveled along the boundary layer's outer edge. Their streamwise velocity component was of several tenths of a percent of the boundary-layer edge velocity $C_{\mathrm{e}}$. 


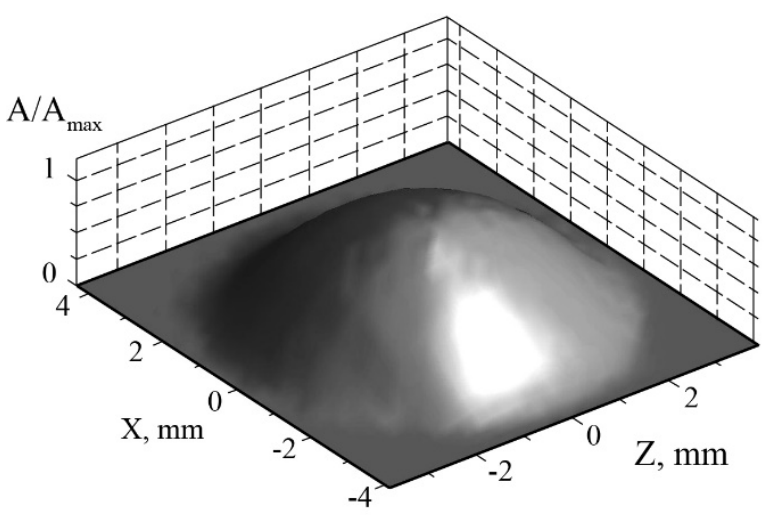

(a)

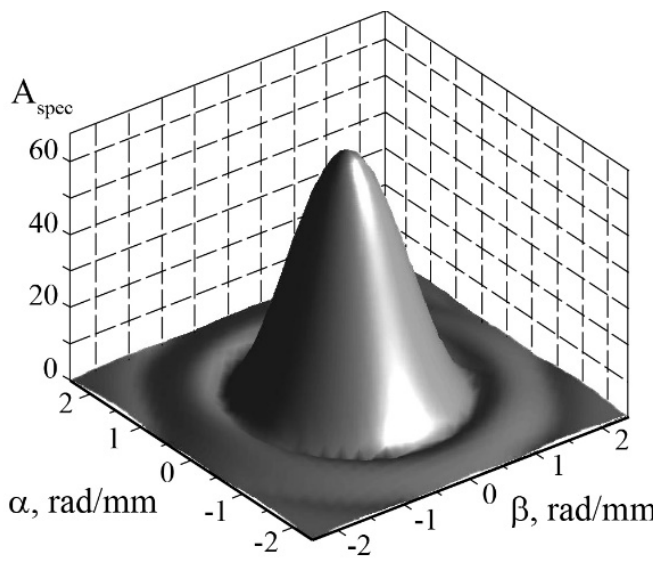

(b)

FIGURE 2. Shape of controlled surface vibrator $(a)$ and amplitude part of its Fourier spectrum $(b)$. Here $\alpha$ and $\beta$ are streamwise and spanwise wavenumbers, respectively

The wave-trains of 3D TS-waves were generated in every studied regime at frequencies of: (i) surface vibrations $f_{\mathrm{s}}$ and the two combination frequencies (ii), i.e. the sum of both, i.e. $f_{+}=f_{\mathrm{s}}+f_{\mathrm{v}}$ and (iii) the difference between both, i.e. $f_{-}=f_{\mathrm{s}}-f_{\mathrm{v}}$. The excitation of the modes with frequency $f_{\mathrm{s}}$ occurred due to the "vibrational" receptivity mechanism, while the sum and difference modes were excited in the boundary layer due to scattering of the controlled freestream vortices at the controlled surface vibrations mentioned above and were associated with the vortex-vibrational receptivity mechanism under study. The measurements were carried out in 15 regimes, four of which (henceforth called main regimes) are discussed in the present paper and indicated in Table 1. The disturbance frequencies $f_{\mathrm{s}}$ and $f_{\mathrm{v}}$ were chosen in a way to provide either downstream amplification or a weak decay of the excited TS-waves. The frequency selection was based on some preliminary linear stability calculations.

TABLE 1. Studied frequency regimes. The frequencies are in $\mathrm{Hz}$.

\begin{tabular}{ccccc}
\hline Regime & $\boldsymbol{f}_{\mathbf{s}}$ & $\boldsymbol{f}_{\mathbf{v}}$ & $\boldsymbol{f}_{-}$ & $\boldsymbol{f}_{+}$ \\
\hline Regime 1_A10 & 221 & 65 & 156 & 286 \\
Regime 2_A10 & 255 & 65 & 190 & 320 \\
Regime 3_A10 & 177 & 53 & 124 & 230 \\
Regime 4_A10 & 98 & 96 & 2 & 194 \\
\hline
\end{tabular}

In the present experiments, it was convenient to use the following spatial coordinates: $x_{\mathrm{s}}^{\prime}$ denotes the arclength in chordwise direction along the curved surface with its origin located at the airfoil leading edge; $z^{\prime}$ is parallel to the airfoil leading edge spanwise axis. The region of the main measurements was located at $x_{\mathrm{s}}^{\prime}=171 \div 401 \mathrm{~mm}$, while the surface disturbance source position was at $x_{\mathrm{so}}^{\prime}=132 \mathrm{~mm}$.

\section{PROPERTIES OF BOUNDARY LAYER DISTURBANCES}

Rather effective excitation of TS-waves was obtained almost in all studied regimes (except for the case of $f_{-}=2 \mathrm{~Hz}$ ). An example of typical spanwise distributions of disturbance amplitudes and phases measured in the boundary layer at a wall-normal distance corresponding to the amplitude maxima of TS-waves (i.e. at $U / U_{\mathrm{e}}=0.435$, were $U$ and $U_{\mathrm{e}}$ denote the local- and boundary-layer-edge streamwise velocity components, respectively) is shown in Fig. 3 (1). Similar spanwise profiles were measured in a number of $x_{\mathrm{s}}^{\prime}$ coordinates. The data obtained in an additional regime, in which the amplitude of surface vibrations was reduced by a factor of $K=2.3$, are indicated by blue diamonds ( 2 in Fig. 3). These additional data being scaled (3 in Fig. 3) practically coincide with the distributions obtained in the ordinary amplitude regime demonstrating the independence of the obtained results from the amplitude of surface vibrations and the linearity of the receptivity mechanism under study. 


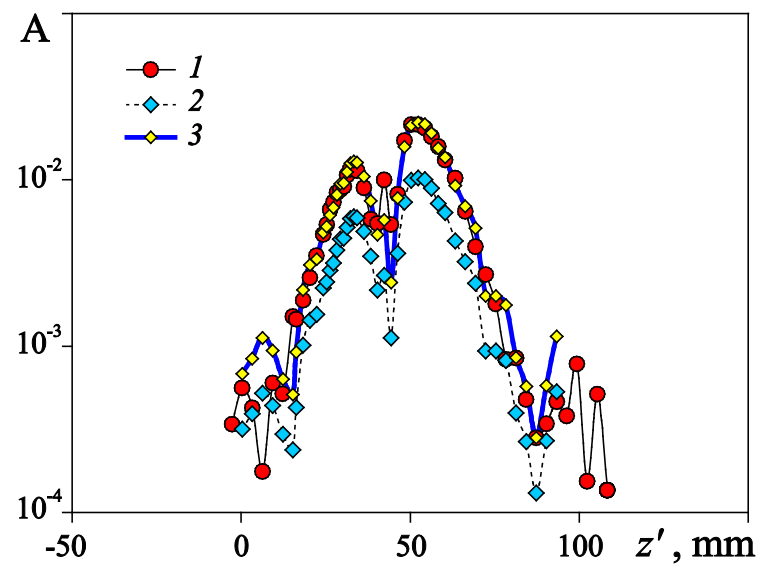

(a)

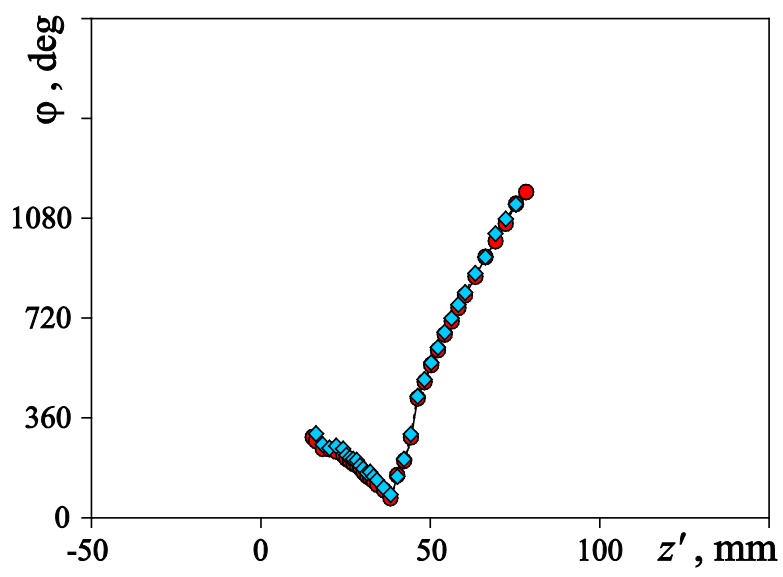

(b)

FIGURE 3. Spanwise profiles of amplitudes $(a)$ and phases $(b)$ of excited TS-waves measured in: 1 - ordinary regime of excitation, 2 - additional regime with amplitude of surface vibrations reduced by a factor of $K=2.3$, and 3 - distributions measured in the additional regime but scaled by a factor of $K . f_{-}=156 \mathrm{~Hz}, x_{\mathrm{s}}^{\prime}=210 \mathrm{~mm}, U / U_{\mathrm{e}}=0.435$

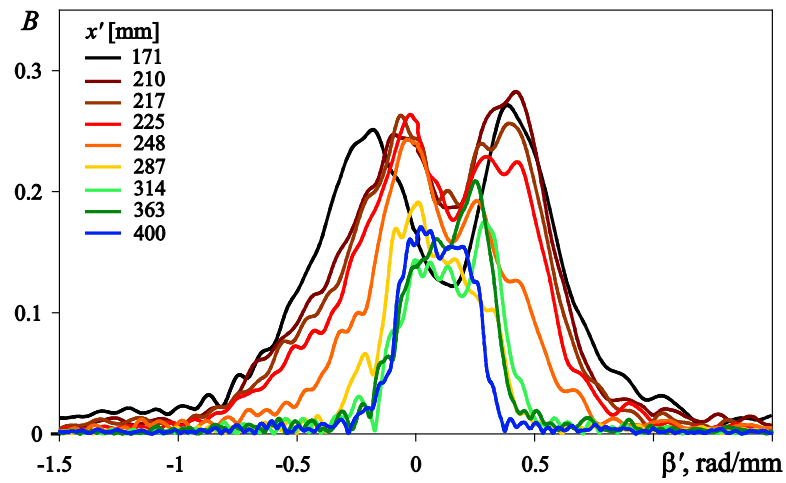

(a)

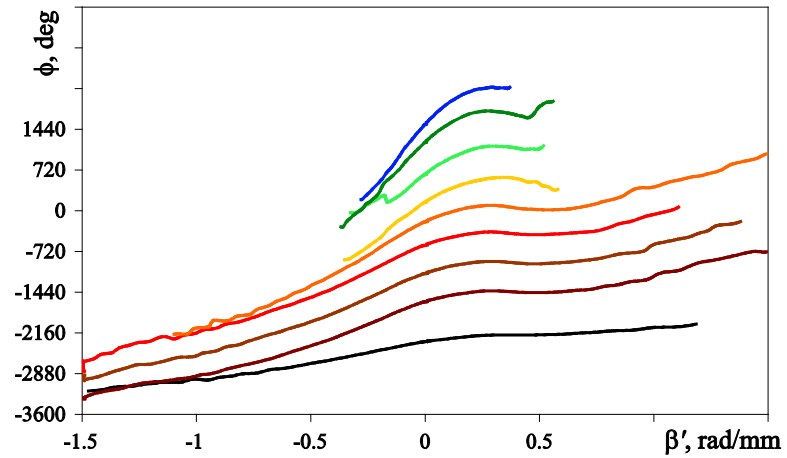

(b)

FIGURE 4. Spanwise-wavenumber spectra of amplitudes and phases of wave-trains of TS-instability modes. Regime 3_A10, $f_{+}=230 \mathrm{~Hz}, U / U_{\mathrm{e}}=0.435$

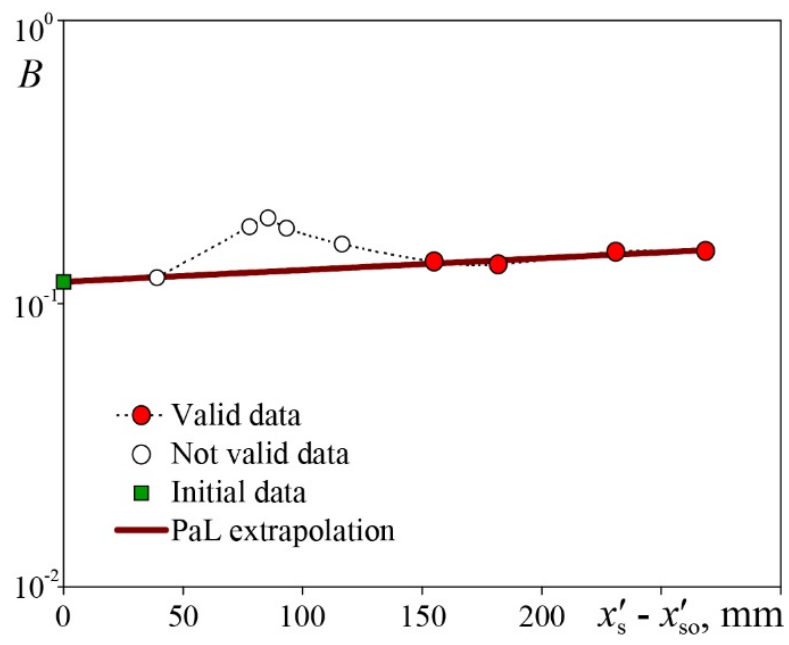

(a)

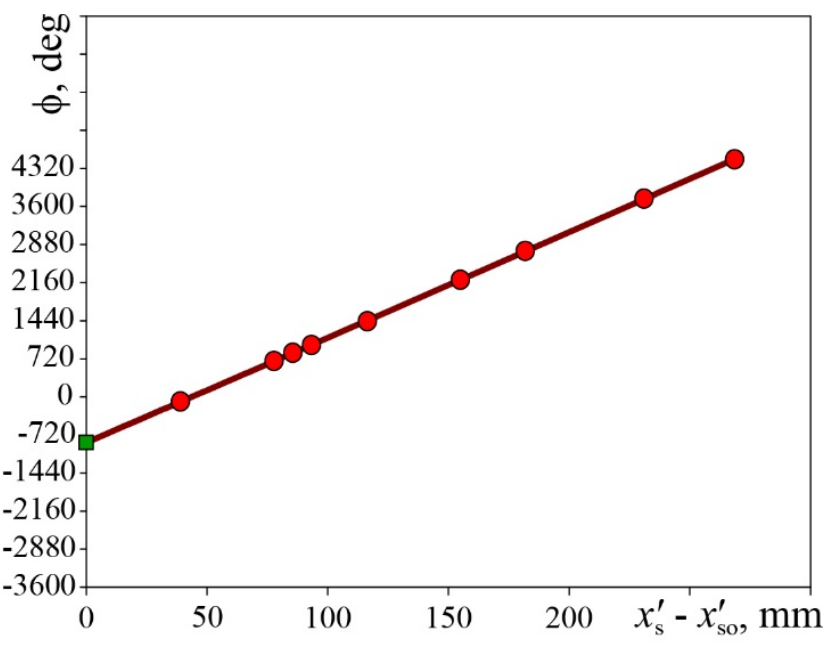

(b)

FIGURE 5. Streamwise distributions of amplitudes $(a)$ and phases $(b)$ of TS waves excited at combination frequency $f_{+}=230 \mathrm{~Hz}$ and their upstream extrapolations by PaL-procedure. Regime 3_A10, $\beta^{\prime}=0.131 \mathrm{rad} / \mathrm{mm}$ 
Examples of spanwise-wavenumber spectra of the boundary layer disturbances are presented in Fig. 4. It is seen that, similar to the 2D Blasius boundary layer case, the spectral amplitudes have a quasi-symmetric shape. However, the symmetry is absent and the fastest growing TS-modes are located not around spanwise wavenumbers $\beta^{\prime}=0$ but somewhat shifted to the vicinity of $\beta^{\prime} \approx+0.1 \mathrm{rad} / \mathrm{mm}$. Spectral phases grow basically with $\beta^{\prime}$.

An example of streamwise distributions of spectral amplitudes and phases of the excited TS-waves is shown in Fig. 5. Filled circles designate the data obtained in a far field of the disturbance source, while open circles correspond to the data displaying admixture of non-modal disturbances in the near-field of the source. Comparison with the corresponding amplitude and phase distributions obtained for the TS-waves excited at frequency $f_{\mathrm{s}}$ by the surface vibrations only (due to action of the vibrational receptivity mechanism) has shown a good correlation with the discussed experimental data obtained in case when $f_{\mathrm{s}}=f_{-}$[12]. This agreement displays the absence of the mechanism of distributed scattering of freestream vortices (with frequency of $f_{\mathrm{v}}$ ) on boundary-layer disturbances excited by surface vibrations at frequency $f_{\mathrm{s}}$ found previously for the case of excitation of CF-instability modes [13].

\section{ESTIMATION OF RECEPTIVITY COEFFICIENTS}

The excitation of TS-waves by a vortex-vibrational receptivity mechanism can be characterized quantitatively by the so-called receptivity coefficients (see $[8,9]$ for instance):

$$
\bar{G}_{s v}\left(f_{ \pm}, \beta^{\prime}\right)=\frac{\bar{B}_{o}\left(f_{ \pm}, \alpha_{r}, \beta^{\prime}\right)}{\bar{C}\left(f_{ \pm}, \alpha_{r s v}, \beta^{\prime}\right) \bar{B}_{v}\left(f_{v}, \alpha_{r v}\right)}
$$

Here $\bar{G}_{\mathrm{sv}}$ is the complex receptivity coefficient for the excitation of TS-modes associated with the vortexvibrational receptivity mechanism, $\bar{B}_{\mathrm{o}}$ denotes the "initial" complex amplitude of excited TS-waves (defined at the position of the surface vibrator), $\bar{B}_{\mathrm{v}}$ is the complex amplitude of the controlled freestream vortices measured at the boundary layer edge at $x_{\mathrm{s}}^{\prime}=x_{\mathrm{so}}^{\prime}$, and $\bar{C}$ is the complex "resonant" Fourier-spectrum of the surface vibrations (i.e. the spectrum of those modes, which have the same spanwise wavenumber $\beta^{\prime}$ as that of the excited TS-instability modes and the streamwise wavenumbers $\left(\alpha_{\mathrm{rsv}}\right)$ satisfying to the resonance condition $\alpha_{\mathrm{rsv}}=\alpha_{\mathrm{r}}+\alpha_{\mathrm{rv}}$, where $\alpha_{\mathrm{r}}$ and $\alpha_{\mathrm{rv}}$ are the streamwise wavenumbers of the TS-waves and freestream vortices, respectively, measured at the position of the surface vibrator).

Since the shape of the membrane and the values of the streamwise wavenumbers $\alpha_{\mathrm{r}}$ and $\alpha_{\mathrm{rv}}$ were carefully measured experimentally, it was not difficult to find the function $\bar{C}$. The values of $\bar{B}_{\mathrm{v}}$ were measured as well, while it was impossible to measure $\bar{B}_{\text {o }}$ directly due to presence of the so-called disturbance source "near-field" (see Fig. 5). These data can be obtained through upstream extrapolation of the experimental streamwise distributions of the disturbance phases and of the logarithms of disturbance amplitudes to the position of the surface vibrator. In the present study, this extrapolation was carried out by means of a well-tested PaL-procedure (by a special combined polynomial function [14]). An example of such extrapolations is shown in Fig. 5. The corresponding extrapolated initial values are shown with green squares.

An example of estimated amplitudes $\left(G_{\mathrm{sv}}\right)$ and phases $\left(\lambda_{\mathrm{sv}}\right)$ of the vortex-vibrational receptivity coefficients is shown in Fig. 6 versus spanwise wavenumber $\beta^{\prime}$ for combination frequency $f_{+}=230$ of Regime 3_A10 (see Table 1). Typical values of the estimated receptivity amplitudes (Fig. 6, a) are of the same order of magnitude as those estimated in experiments [6] for difference TS-mode $\left(f_{-}\right)$excited in the Blasius boundary layer but are lower by a factor of 3 to 4 . There are three possible reasons for this difference: (i) distinction of the receptivity coefficients for difference $\left(f_{-}\right)$and sum $\left(f_{+}\right)$modes (however, they were rather close to each other in the present study), (ii) distinction of the three-dimensional swept-wing boundary layer from the 2D Blasius one, and (iii) inaccuracy of the procedures of PaL-extrapolation in the present case or used in [6]. To increase the quality of estimation of values of the receptivity coefficients, the calculations based on linear stability theory can be used for the experimental data extrapolation mentioned above; our research team is currently working on this problem. Meanwhile, the overall shape of the spanwise distributions of receptivity coefficients observed in the present case of the 3D boundary layer on a swept wing looks rather similar to that found in [6] for the Blasius flow, although the receptivity amplitude minimum is shifted now from the zero spanwise wavenumber to $\beta^{\prime} \approx 0.2 \mathrm{rad} / \mathrm{mm}$ (Fig. 6, a, compare also with Fig. 4 , a). The estimated values of the receptivity coefficients provide us with the possibility of comparison of the 
effectiveness of the vibrational and vortex-vibrational receptivity mechanisms at excitation TS-waves in the swept wing boundary layer. Such comparison has been performed in the present experiments at a fixed level of the freestream vortex amplitude. It turned out that in the case when the amplitude of the streamwise velocity component of freestream perturbations is equal to $1 \%$ of $U_{\mathrm{e}}$, the localized surface vibrations excite TS-modes with initial amplitudes, which are greater by 1-2 orders of magnitude than those excited by the mechanism of scattering of freestream vortices on these vibrations.

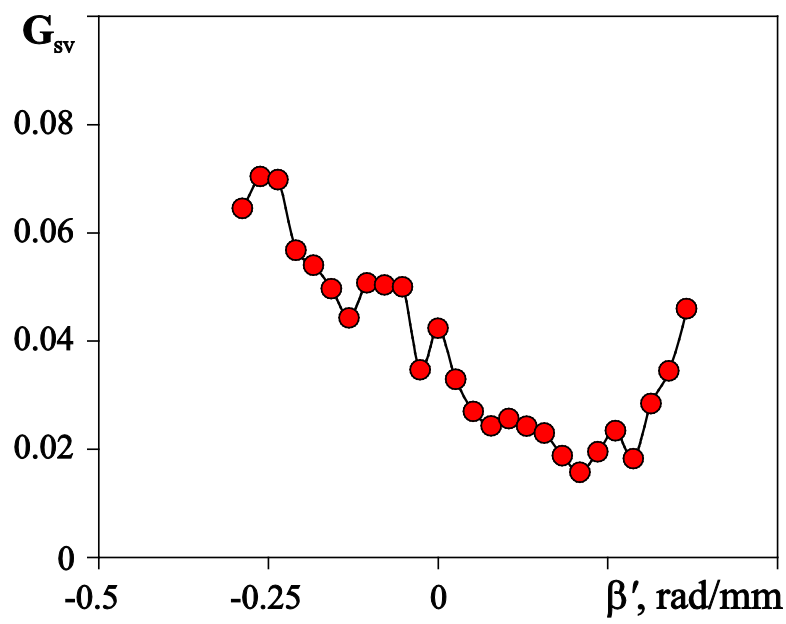

(a)

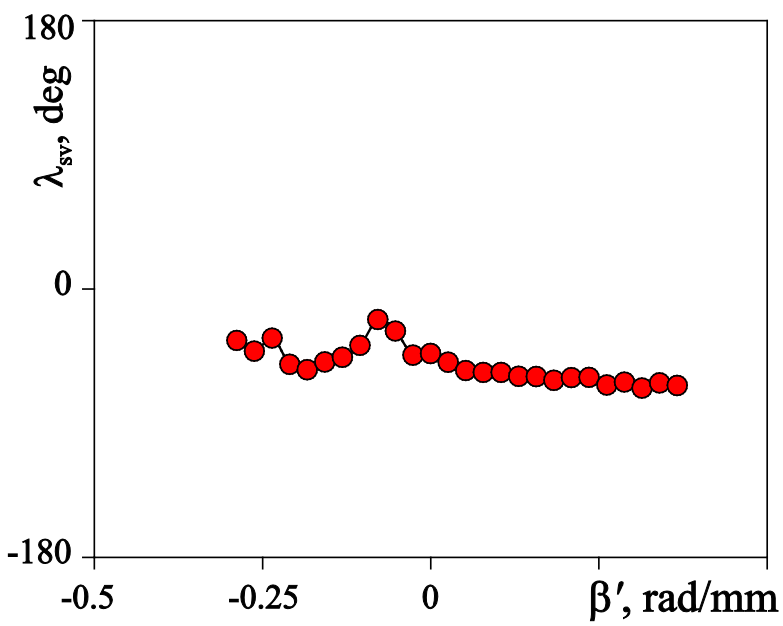

(b)

FIGURE 6. Estimated amplitudes (a) and phases (b) of vortex-vibrational receptivity coefficients for excitation of TS-waves. Regime 3_A10, $f_{+}=230 \mathrm{~Hz}$

\section{ACKNOWLEDGMENTS}

The work reported here has been carried out within "RECEPT" project of the EU Seventh Framework Programme, grant No ACPO-GA-2010-265094 and (partly) within the framework of the Program of Fundamental Scientific Research of the state academies of sciences in 2013-2020 (project No. AAAA-A17-117030610128-8). The experimental study includes a significant contribution of P.H. Alfredsson, N. Tillmark, and A. Segalini.

\section{REFERENCES}

1. Y. S. Kachanov, V. V. Kozlov, and V. Y. Levchenko, Fluid Dyn. 13, 704-711 (1979).

2. Y. S. Kachanov, V. V. Kozlov, V. Y. Levchenko, and V. P. Maksimov, "Transformation of external disturbances into the boundary layer waves," in Proceedings of the Sixth International Conference on Numerical Methods in Fluid Dyn., edited by H. Cabannes, M. Holt, V. Rusanov (Springer, 1978), pp. 299-307.

3. Y. S. Kachanov, V. V. Kozlov, and V. Y. Levchenko, Origin of Turbulence in Boundary Layer (Nauka, Novosibirsk, 1982). (in Russian)

4. A. J. Dietz, J. Fluid Mech. 378, 291-317 (1999)

5. S. Bake, V.I. Borodulin, Y.S. Kachanov, and A.P. Roschektayev, "Experimental study of 3D localized boundary-layer receptivity to free-stream vortices by means of two-source method" in XI International Conference on Methods of Aerophysical Research. Proceedings. Part I, edited by A.M. Kharitonov (Inst. Theor. \& Appl. Mech., Novosibirsk, 2002), pp. 28-33.

6. V. I. Borodulin, Y. S. Kachanov, A. P. Roschektayev, and S. Bake, Thermophysics and Aeromechanics 11(2), 185-198 (2004).

7. S. Herr, W. Würz, and S. Wagner "Experimental investigation of local vortex roughness receptivity of a boundary layer on an airfoil," in New Results in Numerical and Experimental Fluid Mechanics IV. Notes on Numerical Fluid Mechanics and Multidisciplinary Design, edited by C. Breitsamter et al (Springer, Berlin, Heidelberg. 2004), pp. 87.

8. V. I. Borodulin, A. V. Ivanov, Y. S. Kachanov, and A. P. Roschektayev, J. Fluid Mech. 716, 487-527 (2013). 
9. V. I. Borodulin, A. V. Ivanov, Y. S. Kachanov, and A. P. Roschektayev, J. Fluid Mech. 793, 162-208 (2016).

10. A. V. Ivanov, Y. S. Kachanov, and D. A. Mischenko, Thermophysics and Aeromechanics 21(6), 663-678 (2014)

11. D. G. Romano, P. H. Alfredsson, A. Hanifi, R. Örlü, N. Tillmark, V. I. Borodulin, A. V. Ivanov, Y. S. Kachanov, and M. Minervino, Applied Mechanics and Materials 390, 96-102 (2013).

12. V. I. Borodulin, A. V. Ivanov, Y. S. Kachanov, D. A. Mischenko, R. Örlü, A. Hanifi, and S. Hein, "Excitation of 3D TS-waves in a swept-wing boundary layer by surface vibrations and freestream vortices," in International Conference on the Methods of Aerophysical Research (ICMAR 2018), AIP Conference Proceedings 2027, edited by V. M. Fomin (American Institute of Physics, Melville, NY, 2018), pp. 030136.

13. Y. S. Kachanov, V. I. Borodulin, A. V. Ivanov, D. A. Mischenko, R. Örlü, A. Hanifi, and S. Hein, "Generation of unsteady CF-instability modes by vibrational and vibration-vortex localized receptivity mechanisms," in International Conference on the Methods of Aerophysical Research (ICMAR 2018), AIP Conference Proceedings 2027, edited by V. M. Fomin (American Institute of Physics, Melville, NY, 2018), pp. 020010.

14. V. R. Gaponenko, A. V. Ivanov, Y. S. Kachanov, and J. D. Crouch, J. Fluid Mech. 461, 93-126 (2002). 